\title{
MIDI observations of the nuclear obscuring torus in NGC 1068
}

\author{
Walter Jaffe ${ }^{1}$, Klaus Meisenheimer ${ }^{2}$, \\ Huub Röttgering ${ }^{1}$, Christoph Leinert ${ }^{2}$ and Andrea Richichi ${ }^{3}$ \\ ${ }^{1}$ Leiden Observatory, Neilsbohrweg 2, NL-2333 CA Leiden, Netherlands \\ ${ }^{2}$ Max-Planck-Institut-für-Astronomie, Köningtuhl 17, D-69117 Heidelberg, Germany \\ ${ }^{3}$ European Southern Observatory, Karl Schwartzschile Strasse 2, D-85748 Garching be \\ München, Germany
}

\begin{abstract}
We have taken N-band spectra of the nuclear dust regions of NGC 1068 with the newly commissioned Mid-InfrareD-Interferometer (MIDI), with a spatial resolution of $\sim 10$ milliarcsec $(\sim 1 \mathrm{pc})$. We resolve the near-nuclear emission into a warm component $(\sim 300 \mathrm{~K})$ $2.1 \times 3.4 \mathrm{pc}$ in size and a smaller hot component. We see a strong silicate absorption in front of the central component that differs in form from normal olivine-type profiles. This thick dust structure cannot be supported for the length of the active phase of the AGN by gas pressure or turbulent motions.
\end{abstract}

A parsec sized "obscuring torus" of dust has long be postulated to support unified theories of AGNs (Antonucci 1993, Krolik \& Begelman, 1998) and to explain the infrared SEDs of Seyfert galaxies (Efstathiou et al. 1995, Granato et al. 1997) Such an object has never been imaged, however, in the sense of measurements that directly measure its size, composition, or thermal structure, because for even the nearest Seyfert galaxies this requires a resolution of $\sim 10$ millarcsec (mas) in the near/mid-IR. This is unobtainable with any current earth- or space-based single telescopes.

The MIDI instrument (Leinert et al.2003) operates as a Michelson interferometer, combining the signals from pairs of 8-meter telescopes at the Very Large Telescope (VLT) facility of the European Southern Observatory (ESO) at Cerro Paranal, Chile. Operating in the N-band $(8-14 \mu)$ on baselines of $\sim 100$ meters, MIDI provides a resolution of $\sim 10$ mas at wavelengths where the contribution from warm dust near $300 \mathrm{~K}$ peaks. In the observations described here the interferometric signal was dispersed with a $\mathrm{NaCl}$ prism with a spectral resolution $R \sim 30$.

The first successful extragalactic target with MIDI was the nearby Seyfert 2 galaxy NGC 1068, at a distance of about 14.4 Mpc. The results of observations totalling about 15 minutes on source are shown in Fig. 1, along with the results of a simple simulation, described below. The top plot shows the N-band spectrum of the central 200 mas of NGC 1068 as measured by a single telescope and can be compared with the ISO spectrum of the same region (Lutz et al., 2000. The silicate absorption from 8-13 $\mu$ is visible in both the MIDI and ISO spectra, but the forbidden lines of [Ne II], [Ar III] and [S IV], and the strong PAH features in the ISO data are missing in the MIDI spectra, indicating that they probably arise in star forming regions outside the central 20 pc radius.

The second and third plot show the interferometric spectra from two short measurements with projected baselines of 42 and 78 meters respectively at position angles of - 45 and +2 degrees respectively (the jet axis in NGC 1068 is essentially north-south at p.a. $0)$. The full fringe size for these two baselines was 26 and 49 mas at $\lambda=10 \mu$ scaling as $\lambda$. The effective resolution is about half the fringe size, corresponding to 0.9 and 1.7 
parsec at the distance of NGC 1068. In these plots it is clear that the correlated flux has dropped greatly, indicating that the emitting region is well resolved by the fringes. Also evident is that the relative importance of the silicate absorption feature increases with resolution. This indicates that most of the absorbed flux arises from a region of size equal or smaller to the highest resolution obtained.

Since we measured only two projected baselines, rather than the hundreds routinely measured in radio aperture synthesis observing, we cannot create a true image of the source, but only parameterize its overall structure. We do this by assuming that the emission arises from blackbody components with 2-d gaussian emission profiles whose axes are oriented parallel/perpendicular to the jet axis. We pick the temperature, axes sizes, greybody emissivity, and foreground silicate optical depth to best fit the three observed spectra.

We find that a single blackbody gaussian cannot fit both the short and long wavelength ends of the spectrum. The model has effectively 4 parameters (2 axes, 1 temperature, 1 emissivity) and there are effectively six measured points (two wavelength regions measured with the single telescope and two projected baselines each). Introducing wavelength-dependent emissivity that drops with increasing wavelength, as expected of dust, only makes the fit worse. We are thus forced to a two-component models with two different temperatures. The best fit is a warm component $(T \simeq 320 \mathrm{~K})$ with axes of $3.4 \times 2.1 \mathrm{pc}(\mathrm{FWHP}$, major axis $\perp$ jet$)$ and a hot component $(T>800 \mathrm{~K}) 0.7 \mathrm{pc}$ in size along the jet and $<1 \mathrm{pc}$ across. Mostly likely the warm component represents the bulk of the dust heated to this temperature by the UV/X-ray emission from the central accretion disk. Preliminary radiative transfer modelling indicates that the hot component is probably a narrow inner throat of the dusty material, close to the nucleus and heated to near the dust sublimation temperature. It is noteworthy that the hot component is significantly extended along the jet. Hence torus models whose inner structure is a narrow wedge are not tenable; the hottest regions would be only the tip of the wedge and not resolved along the axis.

In the intermediate spectral region, 9-12 $\mu$ we can investigate the nature of the silicate absorption. The profile in the low resolution spectra resembles that of typical amorphous olivine silicates (e.g. the absorption toward the Galactic Center:Kemper et al.(2004)). The highest resolution spectrum, however, shows a relatively flat profile from 8 to $9 \mu$ and then a sharp drop between 9 and $10 \mu$. We could not fit this profile with the Galactic Center silicate profile. We did find a quite satisfactory fit the the profile of calcium aluminum-silicate $\left(\mathrm{Ca}_{2} \mathrm{Al}_{2} \mathrm{SiO}_{7}\right)$ seen in emission in some supergiants (Speck et al.2000). This material has a higher sublimation temperature than olivine, and may survive in the hottest inner part of the dust system. At this point we cannot exclude other, non-olivine dust composition.

The multiple absorption and re-emission of photons in an optically thick dust system makes direct comparison of its spectrum to that of an optically thin stellar shell somewhat risky. "Warm" photons shortwards of $8 \mu$ might diffuse through the clouds and slowly lose energy, filling in the "missing" flux in the $8-9 \mu$ region. We have, however, modelled this process with a 3 -d radiation transfer code. We find that it is possible to fill in this missing flux, but only if at the same time most of the silicate absorption in the 9-11 $\mu$ region is also filled in. It does not seem possible to create the sharp edge at $9 \mu$ by multiple absorption processes. The peak optical depth toward the hot component is about 2 which corresponds to $A_{V} \sim 40$.

Our initial observations have already given "shape" to a rather abstract conception of an obscuring structure. It is a rather thick body with $h / r \sim 0.6$, at least in this temperature range, 


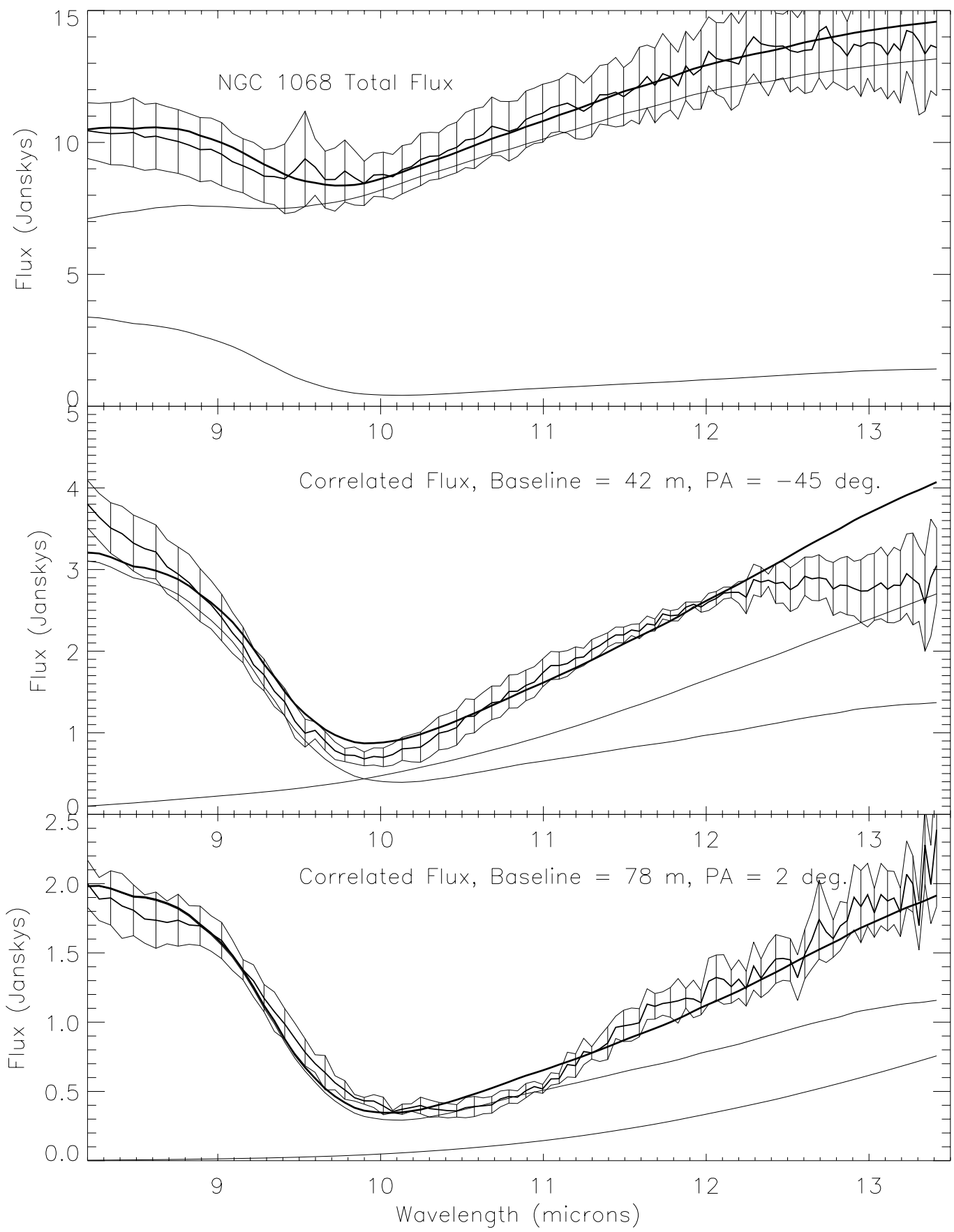

Figure 1. Figure 1. (a) Single Telescope MIDI spectrum of NGC 1068. Here and below the wiggly thin line represents the MIDI measurements, the cross hatching the r.m.s. variations seen between independent measurements, the thick smooth line the model described in the text and the two thinner smooth lines are the contributions by the warm and hot components independently; the hot component dominates at shorter wavelengths. (b) An interferometric spectrum with $42 \mathrm{~m}$ projected baseline taken in November, 2003. (c) a spectrum with $78 \mathrm{~m}$ projected baseline taken in June, 2003. 